\title{
APUNTES DE LA PARTICIPACIÓN CIUDADANA, MODELOS Y HERRAMIENTAS PARA SU GESTIÓN
}

\section{MEMORANDUMS OF THE CITIZEN PARTICIPATION, MODELS AND TOOLS FOR THEIR MANAGEMENT}

\author{
Yudith Salvador Hernández ${ }^{1}$ \\ Mariluz Llanes Font ${ }^{2}$ \\ Reynaldo Velázquez Zaldívar ${ }^{3}$
}

Fecha de recepción: 13 de setiembre de 2017 - Fecha de aceptación: 5 de diciembre de 2017

\begin{abstract}
Resumen
Este artículo se ocupa de recoger los principales modelos y herramientas que como tendencia se han empleado para la gestión de la participación ciudadana, haciendo énfasis en sus aportes y limitaciones. La metodología consiste en identificar y seleccionar los criterios apropiados para la búsqueda de la información; posteriormente recopilar datos sobre los modelos y herramientas de la gestión de la participación ciudadana, teniendo en cuenta el análisis de los componentes principales para la identificar las variables fundamentales. Finalmente, los modelos y herramientas se agrupan por la presencia de variables significativas, que dan cuenta de las tendencias empleadas para lograr gestionar la participación ciudadana formando un conglomerado de elementos que sirven de basamento para que los gobiernos locales orienten sus acciones a tono con la actualidad. En sus conclusiones se destaca que la participación ciudadana no es un elemento fortuito, sino fruto de la gestión de los gobiernos locales; en la medida en que se planifiquen, organicen, lideren y controlen acciones en materia de participación ciudadana, así será su eficacia. Se constata además la tendencia a incluir la gestión por proceso como técnica para gestionar la participación ciudadana por las facilidades que brinda: mapa de procesos, fichas, mapas funcionales y gestión de las interfaces funcionales; aportando significativamente en el correcto desempeño de la participación en los gobiernos locales.

Palabras clave: Administración pública, Tendencias, Ciudadano, Modelos y Herramientas.
\end{abstract}

\begin{abstract}
This article deals with of picking up the principals models and tools that they like tendency have been employed for the administration of the citizen participation, making emphasis in their contributions and limitations. The methodology consist in identify and select of appropriate criterions for the search of the information; next the summary of data about the models and tools of the management of the citizen participation, keeping in mind the analysis of the principal components for the identification of the fundamental variables. Finally the models and tools assembled by the presence of significant variables that they realize the tendencies employed in order to succeed in administering the citizen participation forming a conglomerate of element that they are use as base so that the local governments guide their actions to tone with the present time. In their conclusions stand out that the citizen participation is not a fortuitous element, or else fruit of the administration of the local governments; in the measurement
\end{abstract}

1 Universidad de Holguín, ysalvadorh@uho.edu.cu

2 Universidad de Holguín, mlfon@uho.edu.cu

3 Universidad de Holguín, rector@uho.edu.cu 
that planned, organize, leader and control actions in material of citizen participation, it so will be their efficacy. It ascertains also, the tendency of the inclusion of the management by process like technique in order to management the citizen participation for the eases that offers: map of processes, token, functional maps and management of the functional interface; contributing significantly in the correct performance of the local governments.

Key Words: Public administration, Tendency, Citizen, Models, Tools.

\section{Introducción}

Con la evolución de la humanidad las personas como seres sociales han desempeñado determinados roles que han ido variando según el momento histórico que les ha tocado vivir. La participación ciudadana es considerada uno de estos roles fundamentales del ciudadano como miembro activo dentro de la comunidad a la que pertenece; convirtiéndose en el aspecto neurálgico de los gobiernos actuales. Variadas son las acciones encaminadas a gestionar la participación ciudadana, demandando por tanto identificar los diversos modelos y herramientas empleados actualmente, en aras de obtener un conglomerado de elementos que sirvan de basamento real para orientar cambios necesarios en cualquier gobierno o municipalidad que necesite alinearse con los patrones de comportamiento actuales, con la idea de ser cada día más eficaces en sus acciones.

Significa que los gobiernos deben de acogerse a procederes similares al benchmarking ${ }^{4}$ para lograr implementar técnicas que han sido beneficiosas en sectores similares con el fin de asegurar un poco más el éxito de las reformas a implementar. Utilizar algo que a otros les ha resultado positivo, bajo las mismas condiciones apunta por ser un camino un poco más certero; lo que anima a los responsables del cambio a tomar decisiones con la esperanza del éxito. Esta forma de pensar ha sido legendaria y se ha mantenido a lo largo del tránsito hacia la actualidad, extendiéndose en casi todo el sistema empresarial o privado y claro está, el sector público no escapa a ello y dentro de este los procesos de participación ciudadana.

Conocer qué están haciendo los gobiernos en la actualidad para lograr la eficacia en la participación ciudadana, es el objetivo de esta investigación, con vistas de dotar al lector de los conocimientos básicos y obtener argumentos para comenzar a gestionar la participación ciudadana. En tal sentido se identifican los modelos y herramientas empleados actualmente para la gestión de la participación ciudadana con base en las publicaciones de las bases de datos indexadas en: Thomson, Scopus, Scielo, Latindex y Redalyc; a través del estudio de sus contenidos y las variables significativas, identificando así las tendencias de las diferentes tipologías documentales: artículos, catálogos, tesis doctores y maestrías, correspondiente al período 2000-2015. Después de la poda y la eliminación del solapamiento la cifra se ajustó a 225 registros, utilizándose en la búsqueda los términos participación ciudadana, gestión, modelos y herramientas.

\section{Modelos de participación ciudadana}

Diferentes modelos de participación ciudadana emergen de la bibliografía consultada, se procedió al estudio de 14 de ellos: Jacques (2013); Bleda, Santos y Fernández (2008); Rodríguez (2008), Vitoria (2014); Ministerio Público (2008); Solano (2011); Vázquez y Zamorano (2015); Martínez y Rosende (2011); Guanche (2012); Colpensiones (2013); Urkidi (2013); Colectivo de autores (2015);

4 De acuerdo con Bengt Karlöf y Svante Östblom (1993) que plantean que el benchmarking es un proceso sistemático y continuo para comparar nuestra propia eficiencia, calidad y prácticas con aquellas organizaciones que representan la excelencia; los autores asumen que es el proceso continuo de comparar las acciones de gestión de los procesos de participación ciudadana establecidos en diferentes ámbitos y sectores de las instituciones gubernamentales, con el objetivo de valorar sus resultados innovadores para su posterior implementación. 
Ramírez (2015); González (2016). Todos publicados en la última década, algunos bajo la autoría de municipalidades o gobiernos locales.

Se investigan sus principales aportes y limitaciones, a partir de la presencia o no de ocho variables; derivadas del análisis de contenido a los conceptos y consideradas como las de mayor frecuencia de utilización:

- enfoque por proceso: comprensión y cumplimiento de las necesidades y expectativas de los ciudadanos y ciudadanas en relación a la gestión pública; secuencia detallada y precisa de las diferentes actividades que componen los procesos de participación ciudadana para propiciar la transparencia y coherencia de estos procesos; y mejora con base en mediciones objetivas con el propósito de estimular la democracia participativa, la inclusión social y el bienestar de la población.

- orientación hacia los problemas ciudadanos: es la disposición de las instancias gubernamentales para solucionar los problemas ciudadanos en las políticas públicas formuladas.

- herramientas de análisis estadístico: los procesos de seguimiento a la ejecución y evaluación de las políticas públicas y en general a la gestión pública en todos los ámbitos de las instancias gubernamentales, incluirá la aplicación de herramientas estadísticas de comprobación de la eficacia, eficiencia, efectividad, equidad y calidad de la gestión pública que se evalúa y de la participación en particular.

- eficacia: es la capacidad de alcanzar los objetivos previstos en los procesos de participación ciudadana.

- sistema de indicadores: conjunto de indicadores interrelacionados que miden la gestión de los procesos de participación ciudadana.

- necesidades y expectativas de participación: representan las demandas de intervención de los ciudadanos y ciudadanas en la construcción social de las políticas públicas y en general en la gestión pública de las instancias gubernamentales.

- interfaces funcionales: es el solapamiento que tiene lugar entre las diferentes unidades funcionales involucradas en los procesos de participación ciudadana.

- interacciones significativas: interrelaciones que impactan notablemente en los valores y comportamiento de las variables analizadas en los procesos de participación ciudadana.

El análisis factorial de correspondencia entre las variables, elaborado en el software SPSS confirmó la relación entre ellas, teniendo en cuenta la validez y la fiabilidad asumiendo un valor de 0.78 el Kaiser Meyer Olkin (KMO), denotando la medida de adecuación de las variables analizadas.

Aunque no todos los autores reflejen la inclusión de la mayoría de las variables estudiadas se destacan algunos por sus aportes, al integrar varios conceptos y elementos de gestión:

- Rodríguez (2008) aborda las necesidades y expectativas de participación como una mediación entre la institución y el público.

- Vitoria (2014) introduce explícitamente la necesidad de que los gobiernos desarrollen mecanismos eficaces y desarrollen la participación ciudadana en el ámbito de la gestión municipal.

- Colectivo de autores (2015) del ayuntamiento de Bilbao, aborda herramientas para la gestión de las necesidades y expectativas de participación, y estudios de expectativas ciudadanas, bajo el enfoque de sistema.

- González (2016) propone la transparencia, la rendición de cuenta, la colaboración y la innovación como elementos fundamentales del proceso de PC.

- Vázquez y Zamorano (2015) en el modelo Juárez introduce la creación de organizaciones ciudadanas autónomas que contribuyen a resolver problemas en dicha ciudad y que tienen como principio el fortalecimiento institucional y la vinculación directa con autoridades del gobierno. 
La matriz binaria elaborada da cuentas de la presencia o no de las variables, en los diferentes modelos. Haciendo uso del software SPSS, aplicando el método Ward, se confecciona el dendrograma de la figura 1 donde aparecen los grupos de autores. En un corte en el nivel cinco se denota la presencia de tres grupos: el primero y el más numeroso al contener la mayor cantidad de variables formado por Jacques (2013); Martínez y Rosende (2011); Vitoria (2014); Colpensiones (2013); Guanche (2012); Colectivo de autores (2015); Ramírez (2015) y González (2016); con la presencia de las variables: herramientas de análisis estadístico, sistema de indicadores, interfaces funcionales e interacciones significativas.

El segundo grupo compuesto por: Ministerio Público (2008); Solano (2011) y Bleda, Santos y Fernández (2008), tienen en común las variables enfoque por proceso y, necesidades y expectativas de participación. Por último, el grupo compuesto por Rodríguez (2008); Urkidi (2013); Vázquez y Zamorano (2015) comparte las variables: orientación hacia los problemas ciudadanos y la eficacia.

Figura 1

Análisis de conglomerados de los modelos de participación ciudadana

Ministerio.Publico_2008

Solano.Cornejo_2011

Bleda.Garcia.et.al_2008

Rodriguez.Miren.et.al_2008

Vázquez.y.Zamorano_2015

Urkidi_2013

Jacques_2013

Ramirez.Vivero_2015

Guanche_2012

Martinez.y.Rosende_2011

Ayuntamiento.Victoria.Gasteiz_2014

Colectivo.de.autores_2015

Colpensiones_2013

Gonzalez.Yanez_2016

Dendrograma que utiliza una vinculación de ... Combinación de conglomerados de distancia re-escalados

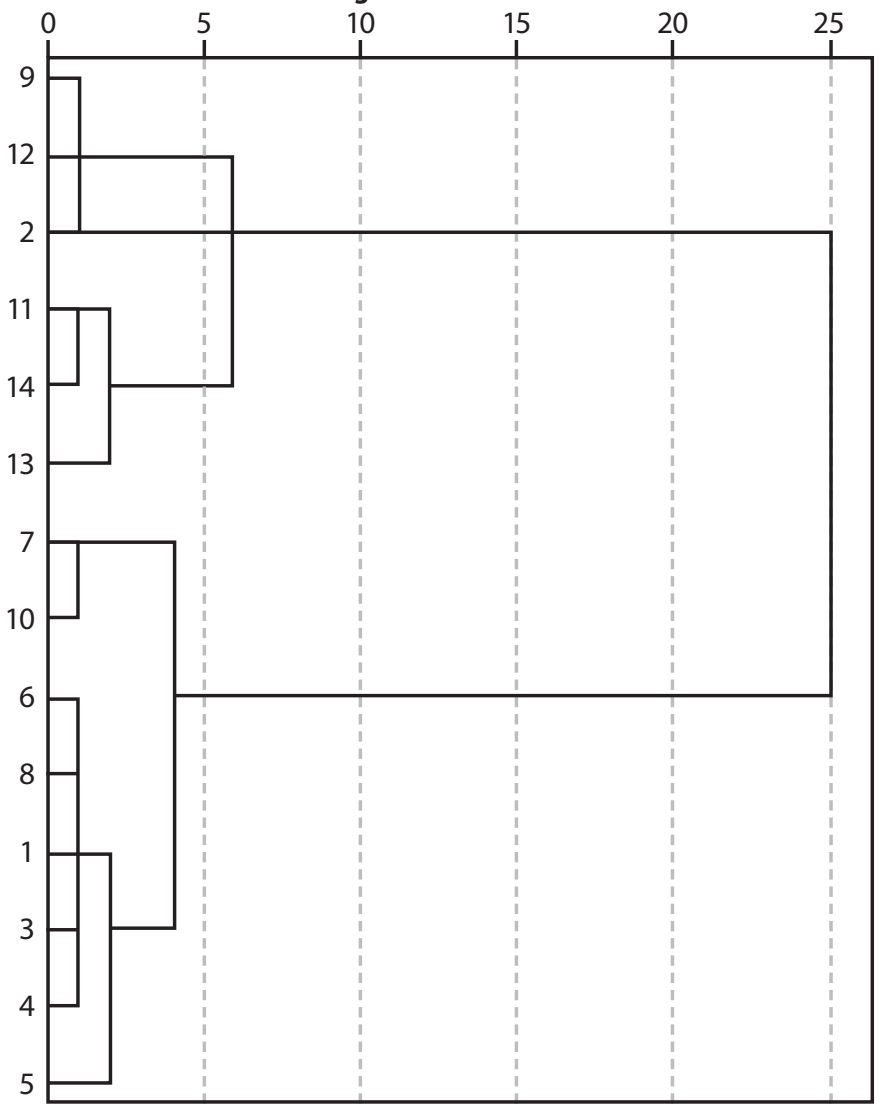


Los modelos que tienen en común las necesidades y expectativas de participación, la abordan unos como medio para lograr la adecuación de las acciones gubernamentales, otros para lograr credibilidad en los gobiernos locales. Los que evidencian de forma explícita la orientación básica de la participación ciudadana hacia los problemas ciudadanos, hacen referencia a la eficacia en dicha soluciones con la intervención de los propios ciudadanos en la generación de ideas contextualizadas a los problemas. La variable enfoque por procesos se visualiza como una técnica para gestionar la participación ciudadana; considerándola útil para la representación de ejes estratégicos y fichas para cada uno de los procesos, haciendo énfasis en al mapa de procesos para la representación gráfica de cada uno de los elementos claves de la participación ciudadana.

Sin embargo, a pesar de la presencia de la variable indicadores, en los modelos se denotan carencias al relacionarse solamente con resultados de encuestas y consultas populares; no siendo así para el diagnóstico de los procesos y control del desempeño de la participación en los gobiernos locales. Se aprecian de manera general otras insuficiencias relacionadas con la orientación hacia las necesidades y expectativas como uno de los ejes centrales de la gestión de la participación ciudadana, criterio que aunque abordado por los autores consultados aún no logran una visión coherente, que contribuya a incrementar la integración de los gobiernos locales.

Así mismo, están latentes incoherencias en la concepción del enfoque por procesos para abordar la participación ciudadana, reconocido solo en el resultado final, lo que limita su utilidad y poco tratamiento a los interfaces funcionales (Llanes, 2015) y a las interacciones significativas entre las partes notorias (figura 2): los problemas (P) y soluciones (S), los procesos de participación (PP) y las necesidades y expectativas de participación ( $\mathrm{N} / \mathrm{E})$; son los vértices fundamentales que forman una trilogía que sostiene o soporta la participación ciudadana y sobre los cuales hay que incidir para desarrollar una adecuada gestión, se traduce en la identificación de los elementos de entrada (problemas) para transformarlos en elementos de salida (soluciones), mediante la adecuación de los procesos de participación y las necesidades y expectativas de participación. El binomio P/S se refiere al equilibrio entre los problemas ciudadanos y sus soluciones efectivas. El estado de equilibrio se alcanza cuando estos elementos funcionan de manera coordinada, a semejanza de imanes, logrando la atracción completa para fusionarse en un todo: la participación ciudadana.

Figura 2

Interacciones significativas

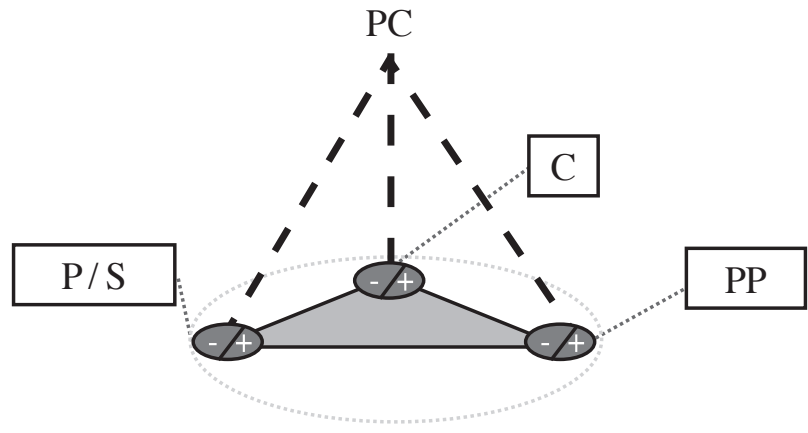

\section{Herramientas para la gestión de la participación ciudadana}

Aparejar las herramientas de participación ciudadana en el ámbito local en función de las necesidades ciudadanas y las potencialidades reales; constituyen nuevas interrogantes para los gobiernos locales. Las descritas en la bibliografía consultada, son muy diversas, con la tendencia de que se han 
trasladado desde el sector empresarial al público y con sus adecuaciones e implementación han logrado sustanciales beneficios en la administración pública y la participación ciudadana.

Se constata la implementación de herramientas de gestión basados en IWA 4/ISO 18091 para gobiernos locales, destacándose autores como: Ayoub, (2008); Losada (2008); Báez (2013); Forbes y Saborío (2015) que consideran que su implementación permite mejorar los niveles de eficiencia, eficacia y efectividad en la gestión de la participación ciudadana. Países como México, Colombia, España dan cuenta de su ejecución para los sectores de salud, educación y gobiernos locales.

La utilización del marco común de evaluación (CAF) ${ }^{5}$ como herramienta para la gestión en la administración pública y la participación ciudadana, según Barroso (2011); Thijs, Stoffels y Heidler (2011) y Molina (2016) es accesible para todas las organizaciones públicas, en especial para que el ciudadano ejerza propiamente una evaluación externa. Ganuza (2010) plantea que esta herramienta convierte a la ciudadanía cada vez más en punto de referencia de la gestión pública y abre las puertas a la participación ciudadana al tener que considerar otras formas de participación y de relación con la ciudadanía.

Seis Sigma es otra herramienta aplicada en la administración pública y en menor grado en la gestión de la participación ciudadana, no obstante se destacan algunos países como: España, Alemania, Países Bajos, Reino Unido, Estados Unidos y autores como: Muñoz (2010); Chiaburu (2014); Duro y Gilart (2016). En América Latina según Morales, Barrera, Rodríguez, Romero, Távara (2014), existen experiencias en la administración pública en Chile, México, Argentina y Perú, fundamentalmente, por su aporte en la aplicación de un amplio abanico de herramientas estadísticas.

Igualmente, el cuadro de mando integral y el control de gestión son herramientas empleadas en la administración pública y en la participación ciudadana, resaltando la posibilidad del alcance de los objetivos y el contacto entre el ciudadano y la administración local. Sobre salen las ideas de Nogueira, Medina, Hernández, Nogueira, Hernández (2009); Bohórquez (2011); Da Fonseca (2015); Espino (2015); Jaquinet (2016) entre otros.

De las herramientas analizadas hasta el momento se destacan algunas limitaciones basadas en las restricciones de la innovación en las organizaciones y en la escasa flexibilidad para el cambio, relacionada con la manera de afrontar la resistencia al cambio. Específicamente, en la aplicación del marco común de evaluación, se denota la necesidad de lograr mayor coherencia y simplicidad a la hora de su aplicación, debiéndose incrementar aspectos de fácil interpretación por los usuarios mediante ejemplos más claros, y ampliar el enfoque de los procesos.

Por su parte, el cuadro de mando integral reporta la mayor parte de los beneficios para determinados grupos dentro de la organización, fundamentalmente, para el grupo directivo que se favorece de los indicadores para el control, no así para el resto; se reconoce que su aplicación es costosa por lo que emprender su uso requiere de un análisis de costo-beneficio a largo plazo. El control de gestión tiene sus limitantes dentro del marco de su aplicación al obedecer en mayor medida a la necesidad del control del presupuesto y su ejecución; la complejidad del sistema público dificulta la separación de responsabilidades entre quienes autorizan, procesan, ejecutan, registran y controlan por lo que su aplicación se complejiza aún más. Asociado a esto se encuentra que los indicadores que se utilizan para medir la eficiencia de los programas suelen ser también costosos.

Como otra de las herramientas analizadas se destaca la gestión por procesos, con una mayor frecuencia de utilización apoyada por numerosos autores: Carreño, Parra y Font (2012); Bocher y Valdés (2013); Pérez (2013); González (2015); Segredo, García, López, León y Perdomo (2015); Molina, (2016); Ricardo (2016); Rivera (2016) como el camino más certero para gestionar la participación ciudadana por las facilidades que brinda: más flexibilidad, rapidez, transparencia e innovación, satisfacción de las necesidades.

$5 \quad$ Herramienta holística que ayuda a las Administraciones Públicas en la búsqueda de la mejora continua presentada en el año 2000 como la primera herramienta europea de gestión de la calidad destinada al sector público. La última versión revisada es el Modelo CAF 2013. (Molina, 2016) 
Siguiendo el precepto de Amozarrain (1999) ${ }^{6}$ de que las organizaciones son tan eficientes como lo son sus procesos; se confirma la causa por la que la gestión por procesos se ha convertido en una de las propensiones mundiales de mayor prevalencia.

Según la Carta Iberoamericana de Calidad en la Gestión Pública, la adopción de una gestión por procesos permite la mejora de las actividades de la administración pública orientada al servicio público y para resultados. La gestión por procesos en la administración pública debe tener en cuenta que el ciudadano es el eje fundamental de la intervención pública, lo cual requiere adoptar enfoques y diseñar los procesos de prestación del servicio desde la perspectiva del ciudadano, usuario o beneficiario. La gestión por procesos implica la identificación de los requerimientos, necesidades y expectativas, de los diferentes destinatarios de las prestaciones y servicios públicos, así como de otros posibles grupos de interés y la definición secuencial detallada y precisa de las diferentes actividades que componen el proceso, para el cumplimiento de los diferentes requerimientos, y en su caso su diagrama.

El análisis y la simplificación de procesos constituyen una herramienta eminentemente práctica para afrontar el cambio en las organizaciones. Ello implica el establecimiento de planes de mejora que permitan obtener resultados en el corto plazo. La puesta en marcha de planes de mejora basados en el análisis y la simplificación de procesos permite alcanzar resultados tangibles en un área concreta y definida, buscando el mínimo de costo en la solución a desarrollar (Galiano, Yánez, Fernández, 2007). De aquí que, el enfoque de procesos sea hoy una herramienta tan poderosa por su capacidad de contribuir de forma sostenida a los resultados (Cordoví, 2013).

\section{Conclusiones}

Se ha constatado la variedad de formas en que se gestiona la participación ciudadana y algunas de sus limitaciones, demostrándose que la participación ciudadana no es un elemento fortuito, sino fruto de la gestión de los gobiernos locales; en la medida en que se planifiquen, organicen, lideren y controlen acciones en materia de participación ciudadana, así será su eficacia.

Los modelos de participación ciudadana muestran la presencia de variables significativas; resaltándose las necesidades y expectativas de participación como uno de los ejes centrales de la gestión de la participación ciudadana; así como la orientación para solucionar los problemas ciudadanos.

El análisis de las herramientas para la gestión da cuenta de la tendencia a la inclusión de la gestión por proceso como técnica para gestionar la participación ciudadana por las facilidades que brinda: mapa de procesos, fichas, mapas funcionales y gestión de las interfaces funcionales; aportando significativamente en el correcto desempeño de la participación en los gobiernos locales. Todos estos elementos, constituyen pautas para que los gobiernos locales promuevan cambios hacia la gestión de la participación ciudadana basados en hechos y experiencias.

\section{Referencias}

Ayoub, José Luis. (2008). Factores de éxito en la implementación de un Gobierno de Calidad: el caso mexicano de la Secretaría de Comunicaciones y Transportes. Enfoques, 9(2), 145-185.

Báez, Luis Germán. (2013). Mejoramiento de la gestión pública con ISO 9001:2008, estudio de caso. Scientia et Technica, 18(1).

Barranquero, Alejandro. (2011). Comunicación alternativa. Debates, escenarios y redes ECOS, 13(1).

Barroso, Julio Manuel. (2011). Europe leading social innovation Social Innovation [Principal innovación social de Europa] Recuperado de http://europa.eu/rapid/ press-release_SPEECH-11-190_en.htm?locale=en

Bengt Karlöf y Svante Östblom (1993). Benchmarking. Alemania: John Wiley \& Sons. Primera Edición

$6 \quad$ Citado por Tamayo (2016) 
Bleda, José María; Santos, Alvaro y Fernández, Cesar (2008). Diseño de un modelo de participación ciudadana en salud en la Comunidad de Castilla La Mancha e implementación del mismo en el Área de Salud de Puerto llano. Rev Adm Sanit. 6(4), 699-714.

Bocher, Laura y Valdés, M. (2013). Understanding business process automation tools for Business Process Management [Entendiendo los procesos de automatización de los negocios según la herramienta de gestión por procesos]. Recuperado de <http:/www.bonitasoft.com/system/files/ documentation_library/bpm_tools_en_13021

Bohórquez, Luz Esperanza. (2011). Sistema de control estratégico y organizacional. Críticas y desafíos. Ciencias Estratégicas, 19 (26), 307-322.

Botero, Luis Horacio y Galvis, Carlos Alberto (2011). Comunicación pública: repensar la comunicación para la democracia. Signo y Pensamiento, 30(1), 334-335.

Calves, Silvio y Sánchez, Barbara Susana (2015). La planificación estratégica gubernamental en la gestión de la administración pública cubana: nuevos escenarios, nuevos aprendizajes. Revista Cubana De Ciencias Económicas-EKOTEMAS, 1(2), 1-11.

Carballal del Rio, Esperanza. (2011). Las estructuras colaborativas. La Habana: Félix Varela, Cuba.

Carreño, Ángela Lorena; Parra, Cecilia y Font, Mabel. (2012). Propuesta metodológica para implementar la gestión pública de la calidad de vida. Ing. Industrial, 33(3).

Chiaburu, George. (2014). Lean Six Sigma en las administraciones públicas. Tesis en opción al título de Master en Empresa y Tecnología de la Información. Universidad de Cantabria. Cantabria. España.

Colectivo de autores (2015). Bilbao y la participación. Recuperado de http://www.bilbaoria2000.org/ ria2000/cas/home/home.aspx

Colpensiones (2013). Modelo de participación ciudadana. Recuperado de http://www.colpensiones.gov. co/publicaciones

Cordoví, Cusa. (2013). La mejora continua a partir de la implantación del sistema Integrado de Gestión en la ECOING 5. Ponencia presentada en el XIII Encuentro TECNOGEST 2013, La Habana, Cuba.

Da Fonseca, Joao (2015). Modelo y procedimiento para el control de Gestión de proyectos de inversión local. Tesis en opción al título de Doctor en Ciencias Técnicas. Universidad de Matanzas Camilo Cienfuegos.

Duro, Viviana y Gilart, Virgilio. (2016). La competitividad en las instituciones de educación superior. Aplicación de filosofías de gestión empresarial: Lean, six sigma y business process management (BPM). Economía y Desarrollo, 157(2).

Espino, Ariel. (2015).Contribución al control de gestión para empresas de Campismo Popular soportado en una plataforma de cambio. Tesis presentada en opción al grado científico de Doctor en Ciencias Técnicas. Universidad Central Marta Abreu de Las Villas, Santa Clara.

Forbes, Roger y Saborío, Milena (2015). ISO 18091: 2014 Guía para la aplicación de la norma ISO 9001:2008 en gobiernos locales. Gestión Municipal. 3.

Galiano, José Antonio; Yánez, Gillermo; Fernández, Emilio. (2007). Análisis y mejora de procesos en organizaciones públicas. Colección de documentos de trabajo. Herramientas para la mejora de la gestión pública. Fundación internacional y para Iberoamérica de Administración y Políticas Públicas.

Ganuza, Ernesto (2010). Nuevos instrumentos de participación: entre la participación y la deliberación. Experiencias internacionales de participación, SaoPaulo, 19-40.

González, Eduardo. (2015). Despliegue de la calidad en la gestión de procesos sustantivos de instituciones de educación superior cubanas. Tesis presentada en opción al grado científico de Doctor en Ciencias Técnicas. Universidad Central Marta Abreu de Las Villas, Santa Clara. 
González, Yanez. (2016). Modelo de gobierno abierto, transparencia, participación ciudadana y colaboración. Curso gobierno digital en la era de la revolución de los datos y la agenda 2030 para desarrollo. Recuperado de http://www.cepal.org

Guanche, Julio Cesar (2012). La participación ciudadana en el Estado cubano. Temas, 70, 69-79.

Hernández, Arialis. (2010). Contribución a la gestión y mejora de procesos en instalaciones hospitalarias del territorio matancero. Tesis presentada en opción al grado científico de Doctor en Ciencias Técnicas, Universidad de Matanzas Camilo Cienfuegos, Matanzas.

ISO 9001. (2015). Sistema de gestión de la calidad. Requisitos.

Jacques, Manuel. (2013). Modelo de participación por afección: un modelo para el desarrollo de la ciudadanía local. Revista Latinoamérica POLIS, 5.

Jaquinet, Rosa Maria. (2016). Contribución al control de gestión en las instituciones de adecuación superior a través de la comunicación organizacional. Tesis en opción al título de Doctor en Ciencias Técnicas. Universidad de Matanzas.

Llanes, Mariluz. (2015) Tecnología para la gestión integrada por procesos de los sistemas normalizados. Aplicación en organizaciones del turismo en Gaviota Holguín. Tesis en opción al grado científico de Doctor en Ciencias Técnicas, Universidad de Holguín Oscar Locero Moya, Holguín.

Losada, Anton. (2008) Democracia, clientes y calidad total en la administración pública. RIPS, 7(2), 39-62.

Martínez, Miguel y Rosende, Silvia. (2011). Participación ciudadana en las agendas 21 locales: cuestiones críticas de la gobernanza urbana. Scripta Nova. Revista Electrónica de Geografía y Ciencias Sociales, 15(355).

Ministerio Público (2008). Guía de la participación ciudadana. Instituto de Estudios del Ministerio Público. Recuperado de http://www.ministeriopúblico

Molina, Pablo. (2016) Proyecto de simplificación administrativa. Revista digital CEMCI. 30-31 (2-3). Recuperado de http://www.cemci.org/revista

Morales, Oswaldo; Barrera, Angel; Rodríguez, Milagros; Romero, Carla; Távara, Rosa. (2014). Modelo de gestión de la innovación para los gobiernos locales del Perú. Serie Gerencia para el Desarrollo. Lima: Universidad ESAN.

Muñoz, Isabel (2010) Aplicación de la metodología de Dirección de Proyectos para la implantación de Lean en el sector sanitario. Tesis en opción al título de Doctor en Ciencias. Universidad de la Rioja. España.

Nogueira, Dianleis; Medina, Alberto; Hernández, Gilberto; Nogueira, Carlos; Hernández, Arialis. (2009). Control de gestión y cuadro de mando integral: énfasis en la perspectiva financiera aplicación en una empresa de servicios de informática. RADM, 44(3). 222-235

Pérez, Wualdo. (2013). Modelo de gestión integrada de la calidad y del medio ambiente en los órganos cubanos de gobierno local. Tesis presentada en opción al grado científico de Doctor en Ciencias Técnicas. Instituto Superior de Tecnologías y Ciencias Aplicadas.

Ramírez, José Miguel. (2015). Modelos de participación ciudadana. Una propuesta integradora. Tesis en opción al grado científico de Doctor en Ciencias Sociales. Universidad Carlos III de Madrid.

Ricardo, Henrry. (2016). Modelo y procedimiento para la gestión y mejora de procesos con la contribución a la integración de sistemas normalizados en Cementeras Cubanas. Tesis en opción al grado científico de Doctor en Ciencias Técnicas. Universidad Central Martha Abreu de las Villas.

Rivera, Natalia Milena. (2016). La participación ciudadana como herramienta de sostenibilidad social en la planificación de infraestructuras. Tesis presentada en opción al título de Master universitario en planificación y gestión en ingeniería civil. Universidad de politécnica de Valencia, Valencia.

Rodríguez, Miren. (2008). Modelos de participación ciudadana como solución a las crisis institucionales motivadas por una oposición social. Análisis, 1(37). 
Rodríguez, Clemencia; Barranquero, Alejandro; Torres, Luis; Krohling, Cicilia (2015). Imágenes contemporáneas de la comunicación ciudadana y comunitaria en América Latina. Revista Internacional de Comunicación y Desarrollo, 1, 13-18.

Segredo, Alina Maria; García, Ana Julia; López, Pedro; León, Pablo; Perdomo, Irene (2015) Enfoque sistémico del clima organizacional y su aplicación en salud pública. Revista Cubana de Salud Pública, 41(1).

Solano, David. (2011). Modelo para participación ciudadana sostenible en los procesos de gestión de riesgos de desastres. XXIV Concurso del CLAD sobre Reforma del Estado y Modernización de la administración pública "Gestión de crisis, emergencias y desastres".

Tamayo, Noris. (2016). Inclusión para el desarrollo. Retos de la administración pública cubana actual. Estudios del Desarrollo Social: Cuba y América Latina, 4 (2), 44-50.

Thijs, Nick; Stoffels, Ann y Heidler, Lena (2011). A diez años del marco Común de Evaluación CAF. Revista Centroamericana de Administración Pública. 60-61, 59-73.

Urkidi, Saioa (2013). La participación en el ámbito local como modelo de construcción de la nueva sociedad. Migraciones. (32), 203-213.

Vazquez, Abraham y Zamorano, Miguel Arturo (2015). Transparencia, rendición de cuentas y gobernanza. La acreditación ciudadana de la función contralora de los municipios en México. Un análisis de la administración 2010-2013 del municipio de Juárez. Revista Legislativa de Estudios Sociales y de Opinión Pública. 8(15), 75-105.

Vitoria-Gasteiz (2014). Revisión modelo participación ciudadana del ayuntamiento de Victoria -Gasteiz. Recuperado de http://www.vixtoria-gasteiz.org 